\section{Prevalência de síndrome metabólica e seus componentes na transição menopáusica: uma revisão sistemática}

\author{
Prevalence of metabolic syndrome and its \\ components in the menopausal transition: \\ a systematic review
}

Karina Giane Mendes 1,2

Heloísa Theodoro ${ }^{3}$

Alice Dalpicolli Rodrigues 3

Maria Teresa Anselmo Olinto 3,4
${ }^{1}$ Centro de Ciências Biológicas e da Saúde, Universidade de Caxias do Sul, Caxias do Sul, Brasil.

2 Programa de Pós-graduação em Endocrinologia,

Universidade Federal do Rio Grande do Sul, Porto Alegre, Brasil.

3 Programa de Pós-graduação em Saúde Coletiva, Universidade do Vale do Rio dos Sinos, São Leopoldo, Brasil.

4 Departamento de Nutrição, Universidade Federal de Ciências da Saúde de Porto Alegre, Porto Alegre, Brasil.

Correspondência M. T. A. Olinto

Programa de Pós-graduação em Saúde Coletiva,

Universidade do Vale do Rio dos Sinos.

Av. Unisinos 950, São Leopoldo RS 93022-000, Brasil.

mtolinto@gmail.com

\section{Abstract}

Metabolic syndrome is a complex disorder involving a combination of cardiovascular risk factors. Menopausal transition can be a key factor in the increased prevalence of metabolic syndrome. The current study aims to evaluate the prevalence of metabolic syndrome and its components in the menopausal transition, using a systematic review. Three reviewers conducted an article search in PubMed. The articles' quality was evaluated according to Strengthening the Reporting of $\mathrm{Ob}$ servational Studies in Epidemiology (STROBE). Based on the selected studies, prevalence of metabolic syndrome increases in the post-menopausal (as compared to pre-menopausal) period, regardless of the population and study design. The change was more significant for waist circumference and blood pressure, suggesting that these components have the greatest influence on prevalence of metabolic syndrome.

Metabolic Syndrome X; Menopause; Post menopause; Premenopause

\section{A síndrome metabólica}

A síndrome metabólica é um transtorno complexo, caracterizado por um agrupamento de fatores de risco cardiovascular.

De acordo com o National Cholesterol Education Program's Adult Treatment Panel III (NCEPATP III) 1, a síndrome metabólica resulta da ocorrência de pelo menos três das cinco desordens, a seguir: obesidade abdominal $(\geq 88 \mathrm{~cm})$, hipertensão arterial ( $\geq 130 \mathrm{mmHg}$ ou $\geq 85 \mathrm{mmHg}$ ), elevação da glicemia ( $\geq 100 \mathrm{mg} / \mathrm{dL}$ ou com diagnóstico de diabetes mellitus), triglicerídeos elevados $(\geq$ $150 \mathrm{mg} / \mathrm{dL}$ ou em tratamento) e redução de colesterol HDL ( $\leq 50 \mathrm{mg} / \mathrm{dL}$ ou em tratamento). Já a Federação Internacional de Diabetes (IDF) enfatiza a presença de obesidade abdominal $(\geq 80 \mathrm{~cm})$ acrescida de mais dois dos componentes já citados para o diagnóstico de síndrome metabólica.

Recentemente, a Associação Americana de Diabetes (ADA) e a Associação Europeia para o Estudo da Diabetes (EASD) publicaram um documento de reflexão sobre a síndrome metabólica, aconselhando uma reorientação sobre os componentes individuais da síndrome, sem considerá-la de forma agregada 2 . Levantaramse várias questões com base em uma crítica aos critérios anteriores da Organização Mundial da Saúde (OMS) e do NCEP-ATP III: (1) Seria de fato uma síndrome, especialmente porque a causa é desconhecida? (2) Teria um propósito útil? (3) Estaria sendo atribuído um "rótulo" (e consequen- 
te medicalização) às pessoas? 2 . Tem-se sugerido que o reconhecimento da síndrome metabólica foi em grande parte impulsionado pela indústria para o desenvolvimento de novos medicamentos 3,4. Mas, segundo o IDF, independentemente das incertezas de definição e etiologia, seria aconselhável considerar a síndrome metabólica como um todo 2 . Alberti et al. 3 defendem que o conceito da síndrome metabólica existe há cerca de 80 anos e a crescente epidemia de diabetes mellitus do tipo 2 e doenças cardiovasculares em todo o mundo, particularmente nos países em desenvolvimento, parecem razões suficientes para identificar e tratar as pessoas com a síndrome.

\section{A síndrome metabólica na transição menopáusica}

Estudos realizados em diferentes populações do mundo revelaram altas prevalências de síndrome metabólica, dependendo do critério diagnóstico empregado e das características da população observada, como gênero, idade, etnia e morbidades associadas, variando as taxas de $8 \%$ a $24 \%$ em homens e de $7 \%$ a $46 \%$ em mulheres 5 . Alguns estudos demonstram que a prevalência de síndrome metabólica aumentou com a idade em ambos os sexos 6,7. No entanto, entre 20 e 50 anos de idade, os homens apresentam uma maior prevalência de síndrome metabólica; a partir dos 50 anos, a prevalência torna-se maior entre as mulheres. Sugere-se que a fase da transição menopáusica possa ser um determinante importante no aumento dessa prevalência 8 .

A transição menopáusica é caracterizada por três períodos, sendo eles: a pré-menopausa, que inicia geralmente aos 40 anos, com redução da fertilidade em mulheres com ciclos menstruais regulares; a perimenopausa, que começa dois anos antes do último ciclo menstrual e estende-se até um ano após, caracterizada por ciclos menstruais irregulares e alterações endócrinas; e a pós-menopausa, que inicia um ano após o último ciclo menstrual 9 .

Alguns autores consideram que a pós-menopausa é um período de hiperandrogenismo relativo como consequência da maior queda de estrogênios, em comparação com os andrógenos, que pode levar à formação da aterosclerose, com aumento dos níveis de colesterol LDL e uma diminuição nos níveis de HDL 10,11,12. De acordo com Janssen et al. 13, mulheres na pósmenopausa possuem maior acúmulo de gordura visceral do que as que ainda menstruam, sendo independente do processo de envelhecimento. O estrogênio é considerado como fator protetor para doenças cardiovasculares nas mulheres pré- menopáusicas, e devido à redução deste hormônio em mulheres após a menopausa ocorre maior suscetibilidade ao aumento de gordura na região abdominal. Outro hormônio sexual relacionado a esse aumento é a testosterona, sendo um forte preditor de gordura visceral e, como tal, associado a um maior risco cardiovascular em mulheres na transição menopáusica.

A temporalidade entre a ocorrência dos componentes da síndrome metabólica e a menopausa tem sido questionada 14 . Não é claro se a ocorrência da menopausa aumenta o risco da síndrome metabólica ou se a idade é que eleva a prevalência. $\mathrm{O}$ aumento da idade estaria relacionado com o processo fisiológico do envelhecimento, principalmente pela redução do metabolismo basal, alteração da composição corporal e estilo de vida inadequado 15,16 .

Considerando que não é clara na literatura científica a relação entre ocorrência de síndrome metabólica e a distribuição dos componentes segundo as alterações hormonais na transição menopáusica, bem como a idade das mulheres, o presente estudo tem como objetivo verificar, por meio de uma revisão sistemática, a prevalência de síndrome metabólica e dos seus componentes na transição menopáusica, ou seja, nas etapas de pré-menopausa, perimenopausa e pós-menopausa.

\section{Materiais e métodos}

\section{Revisão - fase 1}

Foi realizada uma busca em maio de 2011 nas bases de dados: PubMed, LILACS, Science Direct, Cochrane Library, SciELO e Scopus. Os termos usados para busca foram retirados do MeSH (Medical Subject Heading) e DeCS (Descritores em Ciências da Saúde). Os termos usados foram ["Metabolic syndrome X" and "climacteric"] e ["Metabolic syndrome" and "climacteric"] (Tabela 1). A busca nessas bases totalizou 402 documentos. Lendo os títulos, foi observado que 86 estavam repetidos nas diferentes bases de dados, ficando para análise do resumo 316. Desses 316 documentos, foram excluídos estudos especificamente com homens, revisões sistemáticas, ensaios clínicos com animais, ensaios clínicos em laboratórios, livros, resumos de eventos, relatos de caso e outros (consensos, editoriais, guias, correspondência).

Com a retirada desses documentos, restaram 71 (ensaios clínicos com humanos e estudos observacionais: transversal, caso-controle e coorte). Desses, oito foram excluídos por abordarem questões genéticas e disfunções sexuais. 
Número de documentos encontrados na busca.

\begin{tabular}{lcc}
\hline Base de dados & "Metabolic syndrome $X^{\prime \prime}$ and "climacteric" & "Metabolic syndrome" and "climacteric" \\
\hline PubMed & 15 & 31 \\
LILACS & 5 & 16 \\
Science Direct & 12 & 288 \\
Cochrane Library & 2 & 0 \\
SciELO & 1 & 1 \\
Scopus & 14 & 17 \\
\hline
\end{tabular}

Ao final, 63 artigos foram lidos na íntegra para a revisão sistemática. Nessa etapa da busca observou-se que seria necessária a utilização de outros descritores para atingir os objetivos do estudo. Portanto, iniciamos a Revisão-fase 2.

\section{Revisão - fase 2}

Para esta fase da revisão, K.G.M., H.T. e A.D.R. realizaram as buscas, seleção e análise dos artigos e M.T.A.O. fez a revisão final do artigo. O fluxograma da busca está apresentado na Figura 1.

Foi observado na Revisão-fase 1 que a maioria dos artigos elegíveis para serem lidos integralmente estava na base de dados do PubMed. Por esse motivo, os revisores utilizaram somente essa base de dados para a nova pesquisa. Optou-se por analisar somente os artigos encontrados nessa busca.

Os termos usados para descrever a síndrome metabólica foram retirados do MeSH e DeCS. Os termos usados foram metabolic syndrome $x$ or metabolic syndrome and menopause or climacteric or perimenopause or postmenopause or premenopause.

Foram ativados alguns limites na busca: humanos, adultos com mais de 19 anos, feminino; línguas: inglês, espanhol, português; período: últimos 10 anos; exclusão: artigos de revisão e meta-análises.

Utilizando os termos e os limites, 262 artigos foram encontrados.

\section{Seleção dos artigos}

Os títulos e resumos dos 262 artigos foram lidos.

Incluiu-se em uma pré-análise todos os artigos que, pelo resumo, apresentassem resultados relacionados a mulheres na transição menopáusica, com a descrição dos componentes da síndrome e com a divisão dos períodos da transição menopáusica (pré, peri e pós-menopausa). Excluiu-se os artigos em que o objetivo do traba- lho era estudar a síndrome metabólica em uma população que já possuía alguma doença preexistente (câncer, transplantados, HIV, síndrome dos ovários policísticos, síndrome de Cushing, pacientes com distúrbios do sono, diabéticos, doença arterial coronariana, usuários de TRH).

Depois da utilização desses critérios de inclusão e exclusão, restaram 25 artigos para serem lidos na íntegra.

Entre os 25 artigos, foram excluídos oito por não apresentarem a divisão no estado menopáusico, ou o desfecho da síndrome metabólica era referente a outros fatores (adiponectina, transtornos mentais etc.) e em um artigo faltava um componente da síndrome metabólica (hipertensão arterial sistêmica). Assim, restaram 17 artigos para serem avaliados. Desses 17, os revisores selecionaram os artigos conforme os dois objetivos da revisão: a prevalência de síndrome metabólica no estado menopáusico e a análise dos componentes da síndrome metabólica segundo as fases da transição menopáusica.

Para a análise da prevalência da síndrome metabólica, dos 17, quatro artigos foram excluídos, pois só apresentaram a divisão do estado menopáusico dos componentes da síndrome metabólica, e não da prevalência, ou seja, 13 foram selecionados.

Para a análise dos componentes da síndrome metabólica na transição menopáusica, dentre os 17 artigos, seis foram excluídos por não apresentarem os componentes divididos por estado menopáusico, ou seja, 11 foram selecionados para investigar o segundo objetivo do estudo. Entre o total de artigos, sete estiveram incluídos em ambas as análises (Figura 1).

\section{Pontuação do STROBE}

Todos os 17 artigos selecionados foram avaliados pelos critérios do STROBE (Strengthening the Reporting of Observational Studies in Epidemio$\log y$ ) 17 , em que cada um dos 22 critérios recebeu 
Figura 1

Fluxograma da fase 2 da revisão bibliográfica.

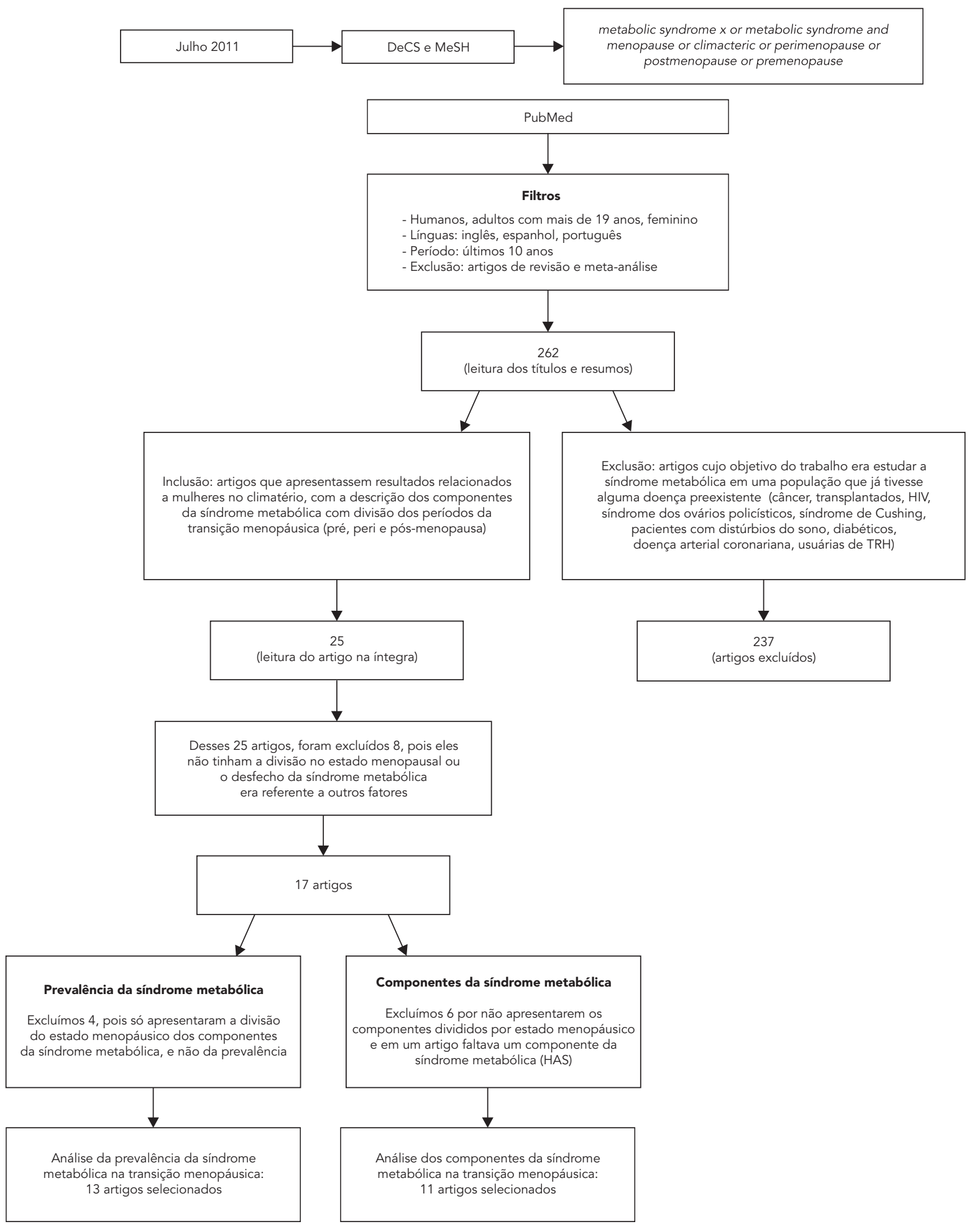


uma pontuação de 0 a 1 . Depois da avaliação de todos os critérios, cada artigo recebeu uma nota de 0 a 22 de cada revisor. Para a nota final, foi realizada uma média das três notas, sendo que a variação das notas entre os revisores não foi superior a 1. De acordo com a nota final, realizou-se a classificação ordinal apresentada na Tabela 2. A pontuação foi transformada em percentual para melhor avaliar a qualidade dos artigos. Os revisores definiram que os artigos que atingissem um percentual superior a $50 \%$ seriam considerados de boa qualidade.

\section{Discutindo os resultados}

\section{Avaliação do STROBE}

Avaliando a Tabela 2, todos os artigos selecionados para a revisão atingiram percentuais maiores que $50 \%$. Observa-se que a maioria dos estudos que atingiram porcentuais acima de $80 \%$ segundo os critérios do STROBE 17 foi os transversais de base populacional e estudos de coorte. Esses foram os estudos que apresentaram as maiores prevalências de síndrome metabólica das mulheres na transição menopáusica, denunciando a magnitude do problema nas mulheres nesta fase da vida.

\section{Prevalência de síndrome metabólica}

Na Tabela 3 encontram-se os artigos nos quais foram apresentadas as prevalências de síndrome metabólica na transição menopáusica. Observase que os estudos que compararam os critérios NCEP e IDF apresentam resultados semelhantes. O IDF mostra prevalências maiores do que o NCEP 18,19,20. Essa diferença ocorre devido ao ponto de corte para a obesidade abdominal - enquanto no NCEP é definido em $88 \mathrm{~cm}$, no IDF o ponto de corte é $80 \mathrm{~cm}$. Logo, devemos interpretar os achados considerando que o critério do NCEP é mais específico, enquanto o IDF torna-se um critério mais sensível.

Entre os estudos longitudinais, em Porto Rico foi encontrada uma prevalência de síndrome metabólica no início do estudo de $23,8 \% 19$. Em pesquisa com mulheres americanas houve diferença em relação à etnia: caucasianas apresentaram $42,3 \%$, seguidas de afro-americanas com $34,6 \%$, sendo as menores incidências em chinesas e japonesas (8,5\% cada) e em hispânicas $(6,2 \%) 14$. Esse resultado está de acordo com os achados nos estudos transversais, nos quais as mulheres asiáticas apresentaram menores prevalências de síndrome metabólica 21,22.

Tabela 2

Pontuação e porcentual de qualidade dos artigos a partir dos critérios do STROBE (Strengthening the Reporting of Observational Studies in Epidemiology).

\begin{tabular}{|c|c|c|c|}
\hline Referência & Tipo de estudo & Pontos & $\%$ \\
\hline Feng et al. 28 & Transversal de base populacional & 20,8 & 94,5 \\
\hline Indhavivadhana et al. 25 & Transversal em ambulatório de climatério & 20,5 & 93,2 \\
\hline Coniglio et al. 18 & Transversal de base populacional & 19,6 & 89,0 \\
\hline Janssen et al. 14 & Coorte & 19,6 & 89,0 \\
\hline Eshtiaghi et al. 33 & Transversal de base populacional & 19,0 & 86,3 \\
\hline Ghosh 34 & Transversal de base populacional & 18,8 & 85,5 \\
\hline Romaguera et al. 19 & Coorte & 18,1 & 82,3 \\
\hline Cho et al. 21 & Transversal em ambulatório clínico & 17,7 & 80,5 \\
\hline Henneman et al. 35 & Coorte & 16,0 & 72,7 \\
\hline Figueiredo Neto et al. 20 & Transversal em ambulatório de climatério & 15,9 & 72,3 \\
\hline Lejsková et al. 42 & Transversal de base populacional & 15,8 & 71,8 \\
\hline Kim et al. 8 & Transversal de base populacional & 15,7 & 71,4 \\
\hline Lin et al. 22 & Transversal em ambulatório clínico & 15,7 & 71,4 \\
\hline Miller et al. 24 & Transversal de base populacional & 14,5 & 65,9 \\
\hline Mesch et al. 26 & Transversal em ambulatório de climatério & 14,3 & 65,0 \\
\hline Ainy et al. 23 & Transversal de base populacional & 14,0 & 63,6 \\
\hline Sieminska et al. 38 & Transversal em ambulatório de ginecologia & 13,0 & 59,1 \\
\hline
\end{tabular}


Tabela 3

Estudos com a prevalência de síndrome metabólica no período da transição menopáusica.

\begin{tabular}{|c|c|c|c|c|c|}
\hline Referência & Desenho & População & Local de estudo & \multicolumn{2}{|c|}{ Prevalência de síndrome metabólica } \\
\hline \multirow[t]{3}{*}{ Lejsková et al. 42} & Transversal & 909 mulheres de & Residentes em Praga & Geral & - \\
\hline & - base & 45 a 54 anos & (República Tcheca) & Pré-menopausa & $22,9 \%( \pm 2,6)$ \\
\hline & populacional & & & Pós-menopausa & $38,3 \%( \pm 4,0)$ \\
\hline \multirow[t]{3}{*}{$\begin{array}{l}\text { Indhavivadhana } \\
\text { et al. } 25\end{array}$} & $\begin{array}{l}\text { Transversal - } \\
\text { ambulatorial }\end{array}$ & $\begin{array}{l}971 \text { mulheres com idade } \\
\text { média de 50,8 anos }\end{array}$ & $\begin{array}{l}\text { Clínica de menopausa } \\
\text { de um hospital }\end{array}$ & Geral & $\begin{array}{c}\text { NCEP: } 15,9 \% \text { (CC de asiáticos) } \\
\text { IDF: } 16 \%\end{array}$ \\
\hline & & & & Perimenopausa & $\begin{array}{c}\text { NCEP: } 12,4 \% \text { (IC: } 9,4-15,4) \\
\text { IDF: } 16 \% \text { (IC: } 15,1-24,3)\end{array}$ \\
\hline & & & & Pós-menopausa & $\begin{array}{l}\text { NCEP: } 16,9 \% \text { (IC: 14,0-19,8) } \\
\text { IDF: } 15,9 \% \text { (IC: 13,6-18,2) }\end{array}$ \\
\hline \multirow[t]{3}{*}{$\begin{array}{l}\text { Figueiredo Neto } \\
\text { et al. } 20\end{array}$} & $\begin{array}{l}\text { Transversal - } \\
\text { ambulatorial }\end{array}$ & $\begin{array}{l}323 \text { pacientes de } 40 \text { a } \\
65 \text { anos, idade média de }\end{array}$ & $\begin{array}{l}\text { Ambulatório de } \\
\text { ginecologia de um }\end{array}$ & Geral & $\begin{array}{l}\text { NCEP: } 34,7 \% \\
\text { IDF: } 49,8 \%\end{array}$ \\
\hline & & 49,7 anos & $\begin{array}{l}\text { hospital público terciário } \\
\text { no Brasil }\end{array}$ & Pré-menopausa & $\begin{array}{l}\text { NCEP: } 24 \% \\
\text { IDF: } 37 \%\end{array}$ \\
\hline & & & & Pós-menopausa & $\begin{array}{l}\text { NCEP: } 44,4 \% \\
\text { IDF: } 61,5 \%\end{array}$ \\
\hline \multirow{4}{*}{$\begin{array}{l}\text { Henneman } \\
\text { et al. } 35\end{array}$} & Coorte & 695 (idade média de 37,8) & Holanda & Geral & - \\
\hline & & na pré-menopausa e 581 & & Pré-menopausa & IDF: $17 \%$ \\
\hline & & (idade média de 60,2) na & & Pós-menopausa & IDF: $43,2 \%$ \\
\hline & & pós-menopausa & & & \\
\hline \multirow{6}{*}{$\begin{array}{l}\text { Romaguera } \\
\text { et al. } 19\end{array}$} & Coorte & 214 mulheres de 36 a 82 & Porto Rico & Geral & NCEP: $23,8 \%$ \\
\hline & & anos acompanhadas por & & & IDF: $32,7 \%$ \\
\hline & & 3 anos & & Pré-menopausa & NCEP: $21,5 \%$ \\
\hline & & & & & IDF: $30,4 \%$ \\
\hline & & & & Pós-menopausa & NCEP: $29 \%$ \\
\hline & & & & & IDF: $45,2 \%$ \\
\hline \multirow[t]{3}{*}{ Eshtiaghi et al. 33} & Transversal & 940 mulheres de & Irã & Geral & NCEP: $26,4 \%$ \\
\hline & - base & 20 a 76 anos & & Pré-menopausa & NCEP $18 \%$ \\
\hline & populacional & & & Pós-menopausa & NCEP: $54 \%$ \\
\hline \multirow[t]{4}{*}{ Coniglio et al. 18} & Transversal & 1.203 mulheres de & Diferentes regiões & Geral & NCEP: $22 \%$ \\
\hline & - base & 40 a 65 anos & geográficas da Argentina & & IDF: $27 \%$ \\
\hline & populacional & & & Pós-menopausa & NCEP: OR 1,61 $(1,18-2,19)$ \\
\hline & & & & & IDF: OR 1,41 (1,06-1,87) \\
\hline \multirow[t]{8}{*}{ Janssen et al. 14} & Coorte & 949 participantes, & Estados Unidos (estudo & Geral & NCEP: $13,7 \%$ (CC de japonesas \\
\hline & & alinhadas na última & SWAN) & & e chinesas $<80 \mathrm{~cm}$ ) \\
\hline & & menstruação, foram & & Perimenopausa & OR $1,45(1,35-1,56)$ [risco por \\
\hline & & recrutadas quando & & & ano de desenvolver síndrome \\
\hline & & estavam na pré ou & & & metabólica na perimenopausa] \\
\hline & & perimenopausa & & Pós-menopausa & OR 1,24 $(1,18-1,30)$ [risco por \\
\hline & & & & & ano de desenvolver síndrome \\
\hline & & & & & metabólica na pós-menopausa] \\
\hline
\end{tabular}

(continua)

Analisando estudos transversais, observa-se que as prevalências em trabalhos de base populacional $(63 \%$ 23; $27,5 \%$ 24) são maiores do que aqueles realizados em ambulatórios (6,2\% 22; 17,9\% 21). As menores prevalências encontradas nos estudos em ambulatórios foram registradas em mulheres asiáticas, o que demonstra uma diferença na pre- valência de síndrome metabólica de acordo com a etnia.

Há um aumento na prevalência de síndrome metabólica de acordo com o estado menopáusico, como observado em estudo transversal de base populacional realizado com iranianas $(53 \%, 54 \%$ e $69 \%$ na pré, peri e pós-menopausa, respectiva- 
Tabela 3 (continuação)

\begin{tabular}{|c|c|c|c|c|c|}
\hline Referência & Desenho & População & Local de estudo & \multicolumn{2}{|c|}{ Prevalência de síndrome metabólica } \\
\hline Cho et al. 21 & $\begin{array}{l}\text { Transversal - } \\
\text { ambulatorial }\end{array}$ & $\begin{array}{c}618 \text { na pré-menopausa } \\
\text { (idade média 40,5) e } 384 \\
\text { na pós-menopausa (idade } \\
\text { média } 59,0 \text { ) }\end{array}$ & $\begin{array}{l}\text { Participantes de exames } \\
\text { anuais no Anam Hospital } \\
\text { em Seul, Coreia do Sul }\end{array}$ & $\begin{array}{c}\text { Geral } \\
\text { Pré-menopausa } \\
\text { Pós-menopausa }\end{array}$ & $\begin{array}{l}\text { NCEP: } 17,9 \% \\
\text { NCEP: } 6,6 \% \\
\text { NCEP: } 35,9 \%\end{array}$ \\
\hline Ainy et al. 23 & $\begin{array}{l}\text { Transversal } \\
\text { - base } \\
\text { populacional }\end{array}$ & $\begin{array}{l}2.182 \text { mulheres, } 537 \\
\text { pré-menopausa (idade } \\
\text { média 47,0), } 311 \text { na } \\
\text { perimenopausa (idade } \\
\text { média 53,0), } 1.334 \text { pós } \\
\text { (idade média 61), de } 45 \text { a } \\
66 \text { anos }\end{array}$ & $\begin{array}{l}\text { Participantes do Tehran } \\
\text { Lipid and Glucose Study } \\
\text { (TLGS) }\end{array}$ & $\begin{array}{l}\text { Geral } \\
\text { Pré-menopausa } \\
\text { Perimenopausa } \\
\text { Pós-menopausa }\end{array}$ & $\begin{array}{l}\text { NCEP: } 63 \% \\
\text { NCEP: } 53 \% \\
\text { NCEP: } 54 \% \\
\text { NCEP: } 69 \%\end{array}$ \\
\hline Lin et al. 22 & $\begin{array}{l}\text { Transversal - } \\
\text { ambulatorial }\end{array}$ & $\begin{array}{c}594 \text { mulheres, } 40 \text { a } 64 \\
\text { anos }\end{array}$ & $\begin{array}{c}\text { Mulheres que } \\
\text { participaram do } \\
\text { levantamento de fatores } \\
\text { de risco para doenças } \\
\text { cardiovasculares em um } \\
\text { centro médico no norte } \\
\text { de Taiwan }\end{array}$ & $\begin{array}{l}\text { Geral } \\
\text { Pré-menopausa } \\
\text { Pós-menopausa }\end{array}$ & $\begin{array}{c}\text { NCEP: } 6,2 \% \\
\text { NCEP modificado: } 8,9 \% \text { (CC } \\
>80 \mathrm{~cm}) \\
\text { NCEP: } 4,2 \% \\
\text { NCEP modificado: } 5,8 \% \text { (CC } \\
>80 \mathrm{~cm}) \\
\text { NCEP: } 9,4\end{array}$ \\
\hline Mesch et al. 26 & $\begin{array}{l}\text { Transversal - } \\
\text { ambulatorial }\end{array}$ & $\begin{array}{c}124 \text { mulheres divididas } \\
\text { em } 4 \text { grupos (pré, } \\
\text { peri-hemorragia, } \\
\text { periamenorreia, pós) }\end{array}$ & $\begin{array}{l}\text { Unidade de climatério na } \\
\text { Divisão de Ginecologia } \\
\text { da Clínica do Hospital da } \\
\text { Universidade de Buenos } \\
\text { Aires (Argentina) }\end{array}$ & $\begin{array}{c}\text { Geral } \\
\text { Pré-menopausa } \\
\text { Perimenopausa } \\
\text { Pós-menopausa }\end{array}$ & $\begin{array}{l}- \\
\text { NCEP: } 0 \% \\
\text { NCEP: } 20-21 \% \\
\text { NCEP: } 22 \%\end{array}$ \\
\hline Miller et al. 24 & $\begin{array}{l}\text { Transversal } \\
\text { - base } \\
\text { populacional }\end{array}$ & $\begin{array}{c}35 \text { na pré-menopausa, } \\
15 \text { na perimenopausa, } \\
116 \text { na pós-menopausa } \\
\text { natural e } 29 \text { menopausa } \\
\text { cirúrgica. } 40 \text { a } 70 \text { anos. } \\
\text { Média de idade: } \\
\text { 57,26 anos }\end{array}$ & $\begin{array}{c}\text { Coorte de mulheres } \\
\text { imigrantes da União } \\
\text { Soviética }\end{array}$ & $\begin{array}{l}\text { Geral } \\
\text { Pré-menopausa } \\
\text { Perimenopausa } \\
\text { Pós-menopausa }\end{array}$ & $\begin{array}{l}\text { NCEP: } 27,5 \% \\
\text { NCEP: } 13 \% \\
\text { NCEP: } 11 \% \\
\text { NCEP: } 68 \%\end{array}$ \\
\hline
\end{tabular}

CC: circunferência da cintura; IC: intervalo de confiança; IDF: Federação Internacional de Diabetes; NCEP: National Cholesterol Education Program; OR: odds ratio; SWAN: The Study of Women's Health Across the Nation.

mente) 23. Porém, pesquisa com imigrantes soviéticas nos Estados Unidos apresentou uma pequena variação entre pré (13\%) e peri (11\%), enquanto a pós-menopausa teve uma prevalência elevada (68\%). Ressalta-se que o número de mulheres na perimenopausa neste trabalho foi reduzido ( $n=15$ ), o que pode ter diminuído o efeito da prevalência da síndrome metabólica na perimenopausa 24 . Entre os transversais em ambulatório, apenas dois artigos apresentaram as prevalências da perimenopausa, as quais assemelham-se aos da pós-menopausa $(12,4 \%$ para $16,9 \% 25 ; 20,5$ para $22 \% 26$ ).

Quanto às comparações realizadas entre pré e pós-menopausa, notou-se um aumento progressivo na prevalência entre estas duas fases. Heterogeneidade étnica, idade, fatores socioeconômicos, estilo de vida, idade da menarca e número de gestações são possíveis fatores que podem influenciar no aumento da prevalência da síndrome metabólica em mulheres 27 . A relação da idade com síndrome metabólica ficou claramente evidenciada. A associação foi diretamente proporcional, sendo que quanto maior a idade, maior a probabilidade da síndrome metabólica, como resultado do processo fisiológico do envelhecimento 28,29,30. Entretanto, em estudo longitudinal realizado nos Estados Unidos, foi encontrado um aumento significativo na prevalência de síndrome metabólica durante a perimenopausa e a pós-menopausa, independentemente da idade e de outros fatores de risco cardiovascular, incluindo ganho de peso e hábito de fumar 14. Essa relação entre idade e síndrome metabólica deve ser objeto de 
outros estudos longitudinais para maiores esclarecimentos sobre esta questão.

\section{Avaliação dos componentes da síndrome metabólica e transição menopáusica}

Na Tabela 4 estão descritos os trabalhos nos quais os componentes da síndrome metabólica no período da transição menopáusica foram analisados separadamente, segundo o desenho de estudo e a população alvo.

\section{Obesidade abdominal}

Durante a transição menopáusica, a forma de distribuição da gordura corporal das mulheres parece se modificar, apresentando tendência de acumular-se na região abdominal 31,32. Com base nos artigos selecionados, verificaram-se elevadas prevalências de obesidade abdominal, sendo que os maiores percentuais foram encontrados nos estudos realizados em ambulatórios. Entre esses, as prevalências mais elevadas foram registradas no trabalho realizado no Nordeste brasileiro, sendo de $76,6 \%$ nas que estavam na pré-menopausa e $85,2 \%$ em mulheres na pós-menopausa 20 . As menores prevalências foram encontradas entre as mulheres asiáticas, que apresentaram 16,4\% na pré-menopausa e 29,1\% na pós-menopausa 22 , sendo que esses dois estudos utilizaram o ponto de corte de $80 \mathrm{~cm}$ para obesidade abdominal.

Já nos trabalhos de base populacional, houve discrepância nos resultados encontrados. Em estudo realizado com 2.671 mulheres coreanas, encontrou-se uma prevalência de obesidade abdominal na pós-menopausa de $67,1 \%$, enquanto o estudo com 940 iranianas apresentou 11,5\%, sendo menor do que a prevalência em mulheres na pré-menopausa $(13,2 \%) 33$. Esse achado pode ser devido à amplitude da faixa etária (20 a 76 anos, média de idade de 33,1) das participantes, que reduziu o efeito da transição menopáusica sobre o acúmulo de gordura central. Quando comparadas as médias de circunferência da cintura (CC) desses estudos de base populacional, a diferença na transição menopáusica é pequena, não ultrapassando $4 \mathrm{~cm}$ entre a pré e a pós-menopausa 23,28,34.

Na transição menopáusica, a pós-menopausa tem apresentado maior prevalência de obesidade abdominal, fato consistente com os estudos analisados. Na Coreia do Sul, o aumento da prevalência de obesidade abdominal na pós-menopausa foi aproximadamente de $40 \%$ em relação às mulheres na pré-menopausa ${ }^{8}$, semelhantemente ao estudo realizado por Cho et al. 21 também na Coreia do Sul, no qual este aumento foi superior a $30 \%$. Na avaliação da média da circunferência da cintura, o estudo de coorte com 1.276 mulheres na Holanda verificou um aumentou na transição menopáusica. Na pré-menopausa a média da circunferência da cintura foi de 78,4cm $( \pm 11,3)$, enquanto que na pós-menopausa foi de $86,1 \mathrm{~cm}$ $( \pm 11,5) 35$.

\section{Elevação da pressão arterial}

Outro critério importante para o diagnóstico de síndrome metabólica é a elevação da pressão arterial que representa um fator de risco independente, linear e contínuo para doenças cardiovasculares 36 . Os valores ótimos para pressão arterial, sistólica e diastólica, são $<120$ e < $80 \mathrm{mmHg}$, respectivamente. Valores a partir de 130 e/ou 85mmHg são considerados alterados, e de $140 \mathrm{e} / \mathrm{ou} 90 \mathrm{mmHg}$ permitem classificar os indivíduos adultos, acima de 18 anos, como hipertensos 37 .

Analisando as prevalências de pressão arterial alterada de mulheres na transição menopáusica, percebeu-se que estas foram mais elevadas na pós do que na pré-menopausa. Entre todos os estudos, aquele realizado no Brasil 20 foi o que apresentou maior prevalência de pressão arterial elevada, tanto na pré $(55,8 \%)$ como na pós-menopausa $(73,4 \%)$. As menores prevalências foram encontradas em ambulatório na Polônia, (7\% na pré e $30 \%$ na pós-menopausa) 38 .

Naqueles trabalhos que apresentaram seus resultados por médias, também se percebeu elevação nos valores da pressão arterial sistólica e diastólica. No estudo de coorte realizado na Holanda 25 e em um transversal de base populacional na Índia 34 as variações mostraram-se semelhantes. A sistólica e a diastólica aumentaram na transição menopáusica, sendo que na pósmenopausa a sistólica apresentou níveis acima de $140 \mathrm{mmHg}$, enquanto a diastólica aumentou na transição, atingindo $87,6 \mathrm{mmHg} 34$. As menores médias de pressão arterial foram encontradas na China 28 e na Argentina 35.

\section{Alteração da glicemia em jejum}

A presença de resistência à ação da insulina tem sido considerada um fator fisiopatogênico importante para a síndrome metabólica 39 . O diabetes mellitus do tipo 2, que apresenta como principal característica a hiperglicemia, é resultado de defeitos na ação da insulina, na secreção de insulina ou em ambos. $\mathrm{O}$ diabetes mellitus do tipo 2 pode ocorrer em qualquer idade, mas é geralmente diagnosticado após os 40 anos, sendo que a maioria dos pacientes apresenta sobrepeso ou obesidade 40. Assim como os outros componentes já avaliados, o aumento da glicemia na 
Componentes da síndrome metabólica na transição menopáusica.

\begin{tabular}{|c|c|c|c|c|c|}
\hline \multirow{2}{*}{$\begin{array}{l}\text { Referência } \\
\text { Figueiredo }\end{array}$} & \multirow{3}{*}{$\begin{array}{c}\text { Desenho } \\
\begin{array}{l}\text { Transversal - } \\
\text { ambulatorial }\end{array}\end{array}$} & \multirow{3}{*}{$\begin{array}{c}\text { População } \\
323 \text { pacientes de } \\
40 \text { a } 65 \text { anos }\end{array}$} & \multirow{2}{*}{$\begin{array}{c}\text { Local de estudo } \\
\text { Brasil }\end{array}$} & \multicolumn{2}{|c|}{$\begin{array}{c}\text { Componentes da síndrome metabólica na } \\
\text { transição menopáusica }\end{array}$} \\
\hline & & & & Pré-menopausa & CC: IDF $76,6 \%$, NCEP $29,9 \%$ \\
\hline \multirow[t]{9}{*}{ Neto et al. 20} & & & & & PA: $55,8 \%$ \\
\hline & & & & & Glicemia: 9,1\% \\
\hline & & & & & Triglicerídeos: 9,1\% \\
\hline & & & & & HDL: $76 \%$ \\
\hline & & & & Pós-menopausa & CC: IDF $85,2 \%$, NCEP $50,9 \%$ \\
\hline & & & & & PA: $73,4 \%$ \\
\hline & & & & & Glicemia: 14,2\% \\
\hline & & & & & Triglicerídeos: $15,4 \%$ \\
\hline & & & & & HDL: $82,8 \%$ \\
\hline \multirow{11}{*}{$\begin{array}{l}\text { Henneman } \\
\text { et al. } 35\end{array}$} & Coorte & 695 na pré-menopausa e & Holanda & Pré-menopausa & CC: IDF $78,4 \mathrm{~cm} \pm 11,3$ \\
\hline & & 581 na pós-menopausa & & & PA diastólica: 76,5 $\pm 9,6$ \\
\hline & & & & & PA sistólica: 126,7 $\pm 15,2$ \\
\hline & & & & & Glicemia: 75,6mg/dL * \\
\hline & & & & & $\begin{array}{l}\text { Triglicerídeos: } 97 \mathrm{mg} / \mathrm{dL} \\
\text { HDL: } 40,2 \mathrm{mg} / \mathrm{dL}\end{array}$ \\
\hline & & & & Pós-menopausa & CC: IDF $86,1 \mathrm{~cm} \pm 11,5$ \\
\hline & & & & & PA diastólica: $80,9 \pm 9,4$ \\
\hline & & & & & PA sistólica: 147,4 \pm 21,8 \\
\hline & & & & & Glicemia: $84,6 \mathrm{mg} / \mathrm{dL}$ * \\
\hline & & & & & Triglicerídeos: $123,9 \mathrm{mg} / \mathrm{dL}$ \\
\hline & & & & & HDL: $40,2 \mathrm{mg} / \mathrm{dL}$ \\
\hline \multirow{12}{*}{$\begin{array}{l}\text { Eshtiaghi et } \\
\text { al. } 33\end{array}$} & Transversal & 940 mulheres de 20 a 76 & Irã & Pré-menopausa & CC: IDF $85,5 \mathrm{~cm} \mathrm{3,2 \%}$ \\
\hline & - base & anos & & & PA diastólica: 9,7\% \\
\hline & populacional & & & & PA sistólica: 15,3\% \\
\hline & & & & & Glicemia: $25,8 \%$ \\
\hline & & & & & Triglicerídeos: $79,4 \%$ \\
\hline & & & & & HDL: $11,2 \%$ \\
\hline & & & & Pós-menopausa & CC: IDF $85,5 \mathrm{~cm} 11,5 \%$ \\
\hline & & & & & PA diastólica: 8,3\% \\
\hline & & & & & PA sistólica: 17,3\% \\
\hline & & & & & Glicemia: 60,3\% \\
\hline & & & & & Triglicerídeos: 95,4\% \\
\hline & & & & & HDL: $13,4 \%$ \\
\hline \multirow[t]{12}{*}{ Ghosh 34} & Transversal & 100 na pré-menopausa e & Índia & Pré-menopausa & CC: $84,8 \mathrm{~cm} \pm 3,2$ \\
\hline & - base & 100 na pós-menopausa & & & PA sistólica: $134,5 \pm 18,0$ \\
\hline & populacional & & & & PA diastólica: $82,4 \pm 13,4$ \\
\hline & & & & & Glicemia: $102,4 \mathrm{mg} / \mathrm{dL} \pm 12,6$ \\
\hline & & & & & Triglicerídeos: $108,2 \mathrm{mg} / \mathrm{dL} \pm 14,4$ \\
\hline & & & & & $\mathrm{HDL}: 45,2 \mathrm{mg} / \mathrm{dL} \pm 4,6$ \\
\hline & & & & Pós-menopausa & CC: $88,6 \mathrm{~cm} \pm 2,8$ \\
\hline & & & & & PA sistólica: $145,0 \pm 17,4$ \\
\hline & & & & & PA diastólica: 87,6 \pm 14,6 \\
\hline & & & & & Glicemia: $123,0 \mathrm{mg} / \mathrm{dL} \pm 14,3$ \\
\hline & & & & & Triglicerídeos: $122,4 \mathrm{mg} / \mathrm{dL} \pm 16,3$ \\
\hline & & & & & $\mathrm{HDL}: 43,6 \mathrm{mg} / \mathrm{dL} \pm 4,2$ \\
\hline
\end{tabular}

(continua) 
Tabela 4 (continuação)

\begin{tabular}{|c|c|c|c|c|c|}
\hline \multirow{2}{*}{$\begin{array}{l}\text { Referência } \\
\text { Cho et al. } 21\end{array}$} & \multirow{2}{*}{$\begin{array}{c}\text { Desenho } \\
\text { Transversal - } \\
\text { ambulatorial }\end{array}$} & \multirow{2}{*}{$\begin{array}{c}\text { População } \\
618 \text { na pré-menopausa e } \\
384 \text { na pós-menopausa }\end{array}$} & \multirow{2}{*}{$\begin{array}{l}\text { Local de estudo } \\
\text { Coreia do Sul }\end{array}$} & \multicolumn{2}{|c|}{$\begin{array}{c}\text { Componentes da síndrome metabólica na } \\
\text { transição menopáusica }\end{array}$} \\
\hline & & & & Pré-menopausa & $\begin{array}{c}\text { CC: } 46,1 \% 80,3 \mathrm{~cm} \pm 7,9 \\
\text { PA: } 6,0 \%\end{array}$ \\
\hline & & & & & PA sistólica: 113,4 \pm 10,7 \\
\hline & & & & & PA diastólica: $69,5 \pm 8,8$ \\
\hline & & & & & Glicemia: $1,9 \% 85,4 \mathrm{mg} / \mathrm{dL} \pm 9,1$ \\
\hline & & & & & Triglicerídeos: $12,3 \%$ \\
\hline & & & & & $96,5 \mathrm{mg} / \mathrm{dL} \pm 55,6$ \\
\hline & & & & & $\mathrm{HDL}: 22,5 \% 60,9 \mathrm{mg} / \mathrm{dL} \pm 13,5$ \\
\hline & & & & Pós-menopausa & CC: $78,9 \% 86,6 \mathrm{~cm} \pm 8,0$ \\
\hline & & & & & PA: $40,6 \%$ \\
\hline & & & & & PA sistólica: $120,9 \pm 10,9$ \\
\hline & & & & & PA diastólica: 76,7 $\pm 9,1$ \\
\hline & & & & & Glicemia: $15,1 \% 93,8 \mathrm{mg} / \mathrm{dL} \pm 17,3$ \\
\hline & & & & & Triglicerídeos: $32,8 \%$ \\
\hline & & & & & $138,1 \mathrm{mg} / \mathrm{dL} \pm 84,4$ \\
\hline & & & & & $\mathrm{HDL}: 37,5 \% 57,1 \mathrm{mg} / \mathrm{dL} \pm 13,5$ \\
\hline \multirow[t]{12}{*}{ Feng et al. 28} & Transversal & 9.097 mulheres & China & Pré-menopausa & CC: $74,4 \mathrm{~cm} \pm 8,1$ \\
\hline & - base & & & & PA sistólica: $117 \pm 17$ \\
\hline & populacional & & & & PA diastólica: $75 \pm 10$ \\
\hline & & & & & Glicemia: 99mg/dL * \\
\hline & & & & & Triglicerídeos: 103mg/dL * \\
\hline & & & & & HDL: $53,7 \mathrm{mg} / \mathrm{dL}$ * \\
\hline & & & & Pós-menopausa & $\mathrm{CC}: 74,5 \mathrm{~cm} \pm 8,3$ \\
\hline & & & & & PA sistólica: $123 \pm 21$ \\
\hline & & & & & PA diastólica: $77 \pm 12$ \\
\hline & & & & & Glicemia: $102,4 \mathrm{mg} / \mathrm{dL}$ * \\
\hline & & & & & Triglicerídeos: $118 \mathrm{mg} / \mathrm{dL}$ * \\
\hline & & & & & HDL: $57,2 \mathrm{mg} / \mathrm{dL}$ * \\
\hline \multirow[t]{19}{*}{ Ainy et al. 23} & Transversal & 2.182 mulheres, 537 & Irã & Pré-menopausa & CC: $92 \mathrm{~cm} \pm 11$ \\
\hline & - base & pré-menopausa, 311 na & & & PA sistólica: $121 \pm 18$ \\
\hline & populacional & perimenopausa e 1.334 na & & & PA diastólica: $80 \pm 10$ \\
\hline & & pós-menopausa & & & Glicemia: 103mg/dL \pm 37 \\
\hline & & & & & Triglicerídeos: 182mg/dL \pm 115 \\
\hline & & & & & $\mathrm{HDL}: 47 \mathrm{mg} / \mathrm{dL} \pm 12$ \\
\hline & & & & Perimenopausa & CC: $94 \mathrm{~cm} \pm 12$ \\
\hline & & & & & PA sistólica: $127 \pm 20$ \\
\hline & & & & & PA diastólica: $82 \pm 11$ \\
\hline & & & & & Glicemia: $106 \mathrm{mg} / \mathrm{dL} \pm 44$ \\
\hline & & & & & Triglicerídeos: $195 \mathrm{mg} / \mathrm{dL} \pm 118$ \\
\hline & & & & & HDL: $46 \mathrm{mg} / \mathrm{dL} \pm 11$ \\
\hline & & & & Pós-menopausa & CC: $72 \% 95 \mathrm{~cm} \pm 11$ \\
\hline & & & & & PA: $69 \%$ \\
\hline & & & & & PA sistólica: $136 \pm 23$ \\
\hline & & & & & PA diastólica: $83 \pm 11$ \\
\hline & & & & & Glicemia: 30\% 114mg/dL \pm 49 \\
\hline & & & & & Triglicerídeos: $67 \% 211 \mathrm{mg} / \mathrm{dL} \pm 133$ \\
\hline & & & & & HDL: $79 \%$ 43mg/dL \pm 11 \\
\hline
\end{tabular}

(continua) 
Tabela 4 (continuação)

\begin{tabular}{|c|c|c|c|c|c|}
\hline \multirow{2}{*}{$\begin{array}{l}\text { Referência } \\
\text { Kim et al. } 8\end{array}$} & \multirow{4}{*}{$\begin{array}{l}\text { Desenho } \\
\text { Transversal } \\
\text { - base } \\
\text { populacional }\end{array}$} & \multirow{4}{*}{$\begin{array}{c}\text { População } \\
\begin{array}{c}1.893 \text { mulheres na pré- } \\
\text { menopausa e } 778 \text { na pós- } \\
\text { menopausa }\end{array}\end{array}$} & \multirow{2}{*}{$\begin{array}{l}\text { Local de estudo } \\
\text { Coreia do Sul }\end{array}$} & \multicolumn{2}{|c|}{$\begin{array}{l}\text { Componentes da síndrome metabólica na } \\
\text { transição menopáusica }\end{array}$} \\
\hline & & & & Pré-menopausa & CC: OMS 28,1\% \\
\hline & & & & & PA: $13,7 \%$ \\
\hline & & & & & Glicemia: 9,8\% \\
\hline & & & & & Triglicerídeos: $18,9 \%$ \\
\hline & & & & & HDL: $54,2 \%$ \\
\hline & & & & Pós-menopausa & CC: OMS 67,1\% \\
\hline & & & & & PA: $63,6 \%$ \\
\hline & & & & & Glicemia: 26\% \\
\hline & & & & & Triglicerídeos: 39,3\% \\
\hline & & & & & HDL: $69,8 \%$ \\
\hline \multirow[t]{10}{*}{ Lin et al. 22} & Transversal - & 594 mulheres & Taiwan & Pré-menopausa & CC: NCEP $80 \mathrm{~cm} 16,4 \%$ \\
\hline & ambulatorial & & & & PA: $19,4 \%$ \\
\hline & & & & & Glicemia: 5,0\% \\
\hline & & & & & Triglicerídeos: 9,2\% \\
\hline & & & & & HDL: $35,6 \%$ \\
\hline & & & & Pós-menopausa & CC: NCEP $80 \mathrm{~cm} \mathrm{29,1 \%}$ \\
\hline & & & & & PA: $37,2 \%$ \\
\hline & & & & & Glicemia: 5,6\% \\
\hline & & & & & Triglicerídeos: $20,5 \%$ \\
\hline & & & & & HDL: $23,9 \%$ \\
\hline \multirow{10}{*}{$\begin{array}{l}\text { Sieminska et } \\
\text { al. } 38\end{array}$} & Transversal - & 56 mulheres na pós- & Polônia & Pré-menopausa & CC: $78,0 \mathrm{~cm}(76,0-85,0)$ \\
\hline & ambulatorial & menopausa e 75 na pré- & & & PA: $7 \%$ \\
\hline & & menopausa & & & Glicemia: 13\% \\
\hline & & & & & Triglicerídeos: $127 \mathrm{mg} / \mathrm{dL}$ * \\
\hline & & & & & HDL: $60,7 \mathrm{mg} / \mathrm{dL}$ * \\
\hline & & & & Pós-menopausa & CC: $89,0 \mathrm{~cm}(84,0-94,5)$ \\
\hline & & & & & PA: $30 \%$ \\
\hline & & & & & Glicemia: 55\% \\
\hline & & & & & Triglicerídeos: $160 \mathrm{mg} / \mathrm{dL}$ * \\
\hline & & & & & HDL: $54,1 \mathrm{mg} / \mathrm{dL}$ * \\
\hline
\end{tabular}

(continua)

transição menopáusica foi observado em todos os estudos.

Os trabalhos que demonstraram maiores prevalências de glicemia em jejum alterada na transição menopáusica foram o de base populacional no Irã ${ }^{33}$, passando de $25,5 \%$ para $60,3 \%$ da pré para a pós-menopausa, e o de ambulatório na Polônia ${ }^{38}$, aumentando de $13 \%$ para $55 \%$ após a ocorrência da menopausa. Entre os estudos ambulatoriais, na Coreia do Sul 21 foi encontrada a menor prevalência de glicemia em jejum na pré-menopausa $(1,9 \%)$, enquanto em Taiwan 22 verificou-se a menor prevalência na pós-menopausa $(5,6 \%)$.

Avaliando os resultados por meio das médias da glicemia em jejum, menores valores foram verificados em estudo de coorte com holandesas 35 , passando de 75,6 para $84,6 \mathrm{mg} / \mathrm{dL}$ da pré para a pós-menopausa. Resultados mais elevados foram observados por estudos de base populacional no Irã 23 e na Índia 34, passando de 103 a 11423 , e de 102 a $123 \mathrm{mg} / \mathrm{dL} 34$, respectivamente, da pré para a pós-menopausa. Entre os estudos transversais realizados em ambulatório, aqueles na Argentina 26 e na Coreia do Sul 21 mostraram as médias de glicemia em jejum, apresentando aumento no período da transição menopáusica, com pequena variação e não ultrapassando o ponto de corte de $100 \mathrm{mg} / \mathrm{dL}$.

\section{Dislipidemias}

Quanto às dislipidemias na transição menopáusica, de acordo com Ferin et al. 41, o hipoestrogenismo associa-se à dislipidemia, pois pode aumentar o colesterol total e o LDL-colesterol, que é aterogênico, por diminuir os receptores hepáticos. 
Tabela 4 (continuação)

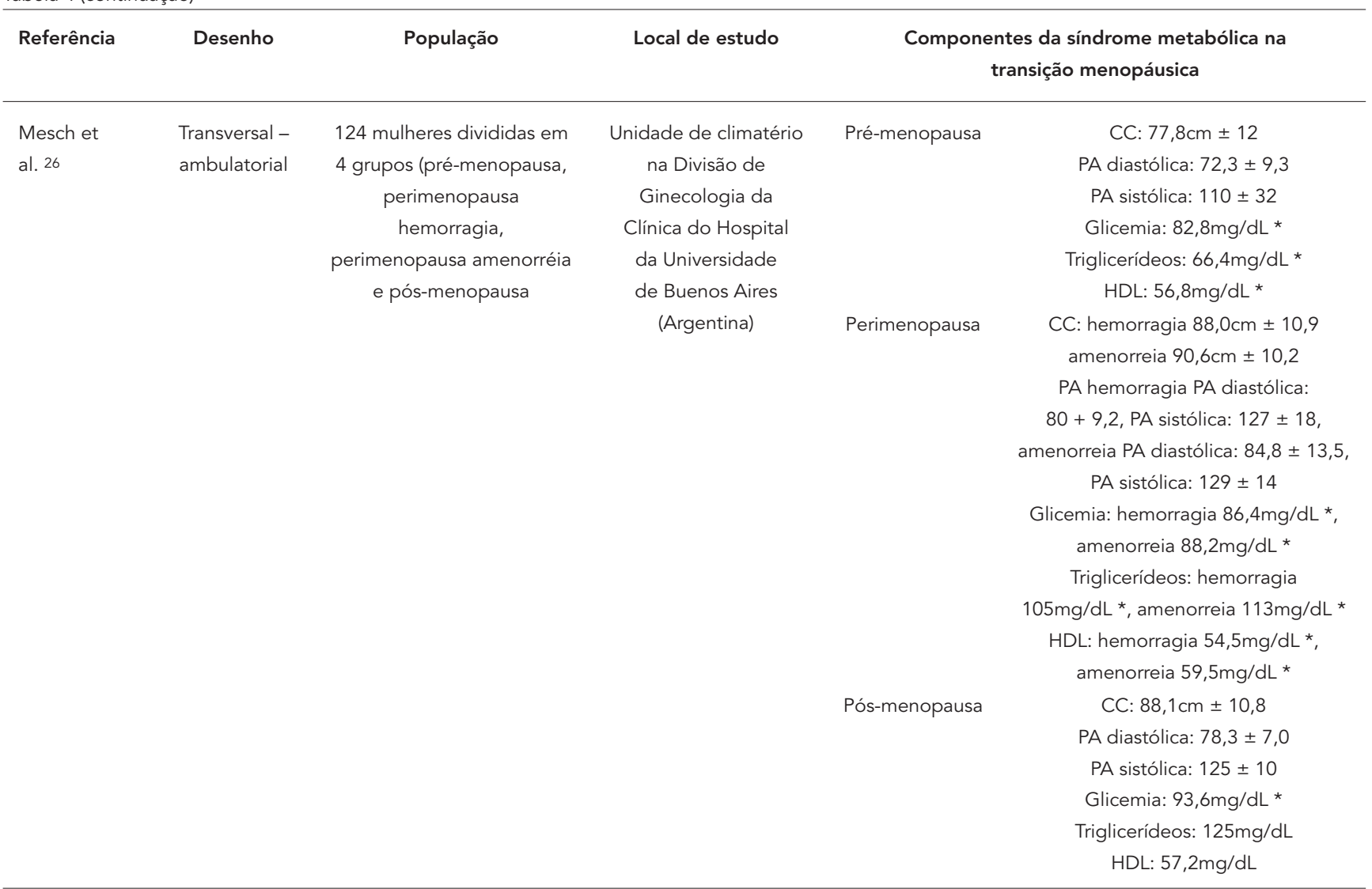

CC: circunferência da cintura; IDF: Federação Internacional de Diabetes; NCEP: National Cholesterol Education Program;

OMS: Organização Mundial da Saúde; PA: pressão arterial.

* Resultados transformados de $\mathrm{mmol}$ em $\mathrm{mg} / \mathrm{dL}$ para comparação de resultados.

Nos artigos selecionados para esta revisão, foi possível observar o aumento dos triglicerídeos na transição menopáusica. Assim como encontrado na análise da prevalência geral de síndrome metabólica, os estudos transversais de base populacional apresentaram maiores prevalências de triglicerídeos aumentados quando comparados aos transversais realizados em ambulatório. Entre os trabalhos que apresentaram as prevalências de triglicerídeos aumentados, o maior porcentual encontrado, tanto na pré quanto na pós-menopausa, foi em um estudo transversal de base populacional realizado com 940 mulheres iranianas de 20 a 76 anos $(79,4 \%$ na pré, $95,4 \%$ na pós-menopausa) ${ }^{33}$. As menores prevalências de triglicerídeos aumentados foram registradas em estudo realizado no Brasil (9,1\% na pré; $15,4 \%$ na pós) 20. Quando os trabalhos apresentaram seus dados por meio de médias, também foi possível notar o aumento no valor dos TG na transição menopáusica. Em estudo no Irã 23, já na pré-menopausa, as mulheres apresentavam um valor médio acima do recomendado $(182 \mathrm{mg} / \mathrm{dL})$, sendo que este valor foi aumentando no período (195 e $211 \mathrm{mg} / \mathrm{dL}$, na peri e na pós, respectivamente). As menores médias foram registradas em estudo transversal de base populacional realizado com chinesas (103mg/dL na pré, 118mg/ dL na pós) 28.

Quanto ao colesterol HDL, a maioria dos trabalhos apresenta uma redução no valor na transição menopáusica, o que não é adequado, pois quanto maiores os valores de HDL, melhor a saúde cardiovascular. As maiores prevalências de HDL abaixo de 50mg/dL foram encontradas no Brasil (76\% na pré, $82,8 \%$ na pós) 20 e na Coreia do Sul (54,2\% na pré, $69,8 \%$ na pós) ${ }^{8}$. As menores prevalências de HDL abaixo de $50 \mathrm{mg} / \mathrm{dL}$ foram registradas em estudo com mulheres iranianas (11,2\% na pré, $13,4 \%$ na pós) 33 . Quando os resultados foram apresentados por meio das médias de HDL, o estudo de coorte que avaliou 1.276 mulheres holandesas mostrou os menores valores, sem diferenças no período da transição menopáusica $(40.21 \mathrm{mg} / \mathrm{dL}$ na pré e na pós-menopausa) ${ }^{35}$. As maiores médias de HDL (60.71 na 
pré, 54,14 na pós) foram encontradas no estudo com polonesas 38 .

\section{Conclusão}

Com base nos estudos analisados, a prevalência de síndrome metabólica aumenta na comparação do período da pré para a pós-menopausa, independentemente da população. Essa tendência foi observada na maioria dos estudos, sem considerarmos o delineamento. As menores prevalências foram encontradas nos estudos realizados com populações asiáticas.

Quanto aos componentes, a maioria dos trabalhos apresentou na transição menopáusica aumento na medida da circunferência da cintura, da pressão arterial, da glicemia em jejum, dos triglicerídeos e redução do HDL. Essa alteração foi mais expressiva nas medidas de circunferência da cintura e pressão arterial. Os estudos com maiores prevalências de síndrome metabólica foram os mesmos que apresentaram as maiores prevalências ou médias de circunferência da cintura e pressão arterial. Sugere-se que esses componentes sejam os que exercem maior influência na prevalência de síndrome metabólica.

Quanto à relação entre síndrome metabólica, estado menopáusico e idade, as conclusões dos estudos apontam para uma maior influência da menopausa na presença da síndrome metabóli- ca. Entre os trabalhos que fizeram a análise dessa relação, a maioria demonstrou que o estado menopáusico foi preditor independente para a síndrome metabólica 8,14,21,24,33. Outros encontraram que o principal fator de risco para o aumento da prevalência de síndrome metabólica foi a idade 20,26.

Para a análise da prevalência da síndrome metabólica e de seus componentes, foram utilizados apenas os artigos encontrados usando-se a busca no PubMed, sem utilizar outras bases de dados ou busca nas referências dos artigos selecionados. Essas podem ser consideradas as limitações deste estudo. É importante destacar que se torna difícil verificar o efeito da menopausa sobre a síndrome metabólica e seus componentes em estudos transversais, visto que ambas as situações sofrem influências de muitos fatores, tais como índice de massa corporal, etnia, classe socioeconômica, atividade física, alimentação e tabagismo.

Destacamos a importância da realização de estudos longitudinais com mulheres desde o início da vida reprodutiva, para maiores esclarecimentos sobre a relação entre a idade, a menopausa, a síndrome metabólica e seus componentes. Trabalhos que considerem as características sociodemográficas e de estilo de vida das mulheres poderão oferecer subsídios para a melhor compreensão dessa relação e contribuir para a proposição de medidas de prevenção.

\section{Resumo}

A sindrome metabólica é um transtorno complexo, caracterizado por um agrupamento de fatores de risco cardiovascular. Sugere-se que a fase da transição menopáusica possa ser um determinante importante no aumento da prevalência da síndrome metabólica. $O$ presente estudo teve como objetivo verificar, por meio de uma revisão sistemática, a prevalência de síndrome metabólica e dos seus componentes na transição menopáusica. Três revisores fizeram a busca dos artigos na base de dados do PubMed. A qualidade dos artigos foi avaliada usando-se o Strengthening the Reporting of Observational Studies in Epidemiology (STROBE).
Com base nos estudos analisados, a prevalência de síndrome metabólica aumenta na comparação do período da pré para a pós-menopausa, independentemente da população e do delineamento do estudo. Quanto aos componentes, a alteração foi mais expressiva nas medidas de circunferência da cintura e pressão arterial. Sugere-se que esses componentes sejam os que exercem maior influência na prevalência de síndrome metabólica.

Síndrome X Metabólica; Menopausa; Pós-menopausa; Perimenopausa; Pré-menopausa 


\section{Colaboradores}

K. G. Mendes participou da concepção e projeto, análise e interpretação dos dados, redação do artigo e aprovação final da versão a ser publicada. A. D. Rodrigues participou da análise e interpretação dos dados, redação do artigo e aprovação final da versão a ser publicada. H. Theodoro contribuiu com o projeto, análise e interpretação dos dados, redação do artigo e aprovação final da versão a ser publicada. M. T. A. Olinto participou da concepção e projeto, revisão crítica relevante do conteúdo intelectual e aprovação final da versão a ser publicada.

\section{Referências}

1. Executive Summary of the Third Report of the National Cholesterol Education Program (NCEP) Expert Panel on Detection, Evaluation, and Treatment of High Blood Cholesterol in Adults (Adult Treatment Panel III). JAMA 2001; 285:2486-97.

2. Duvnjak L, Duvnjak M. The metabolic syndrome - an ongoing story. J Physiol Pharmacol 2009; 60 Suppl 7:19-24.

3. Alberti KG, Zimmet P, Shaw J. The metabolic syndrome: a new worldwide definition. Lancet 2005; 366:1059-62.

4. Reaven GM. The individual components of the metabolic syndrome: is there a raison d'etre? J Am Coll Nutr 2007; 26:191-5.

5. Cameron AJ, Shaw JE, Zimmet PZ. The metabolic syndrome: prevalence in worldwide populations. Endocrinol Metab Clin North Am 2004; 33:351-75.

6. Kim MH, Kim MK, Choi BY, Shin YJ. Prevalence of the metabolic syndrome and its association with cardiovascular diseases in Korea. J Korean Med Sci 2004; 19:195-201.

7. Park HS, Oh SW, Cho SI, Choi WH, Kim YS. The metabolic syndrome and associated lifestyle factors among South Korean adults. Int J Epidemiol 2004; 33:328-36.

8. Kim HM, Park J, Ryu SY, Kim J. The effect of menopause on the metabolic syndrome among Korean women: the Korean National Health and Nutrition Examination Survey, 2001. Diabetes Care 2007; 30:701-6.

9. World Health Organization. Research on the menopause in the 1990. Reports of a WHO scientific group. Geneva: World Health Organization; 1996.
10. Banks AD. Women and heart disease: missed opportunities. J Midwifery Womens Health 2008; 53:430-9.

11. Kalish GM, Barrett-Connor E, Laughlin GA, Gulanski BI. Association of endogenous sex hormones and insulin resistance among postmenopausal women: results from the Postmenopausal Estrogen/Progestin Intervention Trial. J Clin Endocrinol Metab 2003; 88:1646-52.

12. Lee CC, Kasa-Vubu JZ, Supiano MA. Androgenicity and obesity are independently associated with insulin sensitivity in postmenopausal women. Metabolism 2004; 53:507-12.

13. Janssen I, Powell LH, Kazlauskaite R, Dugan SA. Testosterone and visceral fat in midlife women: the Study of Women's Health Across the Nation (SWAN) fat patterning study. Obesity (Silver Spring) 2010; 18:604-10.

14. Janssen I, Powell LH, Crawford S, Lasley B, SuttonTyrrell K. Menopause and the metabolic syndrome: the Study of Women's Health Across the Nation. Arch Intern Med 2008; 168:1568-75.

15. Grundy SM. Multifactorial causation of obesity: implications for prevention. Am J Clin Nutr 1998; 67(3 Suppl):563S-72S.

16. Matsudo S, Matsudo V, Barros T. Impacto do envelhecimento nas variáveis antropométricas, neuromotoras e metabólicas da aptidão física. Rev Bras Ciênc Mov 2000; 8:21-32.

17. von Elm E, Altman DG, Egger M, Pocock SJ, Gotzsche PC, Vandenbroucke JP. The Strengthening the Reporting of Observational Studies in Epidemiology (STROBE) statement: guidelines for reporting observational studies. J Clin Epidemiol 2008; 61:344-9. 
18. Coniglio RI, Nellem J, Gentili R, Sibechi N, Agusti E, Torres M. Metabolic syndrome in employees in Argentina. Medicina (B. Aires) 2009; 69:246-52.

19. Romaguera J, Ortiz AP, Roca FJ, Colon G, Suarez E. Factors associated with metabolic syndrome in a sample of women in Puerto Rico. Menopause 2010; 17:388-92.

20. Figueiredo Neto JA, Figueredo ED, Barbosa JB, Barbosa FF, Costa GR, Nina VJ, et al. Metabolic syndrome and menopause: cross-sectional study in gynecology clinic. Arq Bras Cardiol 2010; 95: 339-45.

21. Cho GJ, Park HT, Shin JH, Kim T, Hur JY, Kim YT, et al. The relationship between reproductive factors and metabolic syndrome in Korean postmenopausal women: Korea National Health and Nutrition Survey 2005. Menopause 2009; 16:998-1003.

22. Lin WY, Yang WS, Lee LT, Chen CY, Liu CS, Lin CC, et al. Insulin resistance, obesity, and metabolic syndrome among non-diabetic pre- and postmenopausal women in North Taiwan. Int J Obes (Lond) 2006; 30:912-7.

23. Ainy E, Mirmiran P, Zahedi Asl S, Azizi F. Prevalence of metabolic syndrome during menopausal transition Tehranian women: Tehran Lipid and Glucose Study (TLGS). Maturitas 2007; 58:150-5.

24. Miller AM, Wilbur J, Chandler PJ, Sorokin O. Cardiovascular disease risk factors and menopausal status in midlife women from the former Soviet Union. Women Health 2003; 38:19-36.

25. Indhavivadhana $S$, Rattanachaiyanont M, Wongvananurak T, Kanboon M, Techatraisak K, Leerasiri $\mathrm{P}$, et al. Predictors for metabolic syndrome in perimenopausal and postmenopausal Thai women. Climacteric 2011; 14:58-65.

26. Mesch VR, Boero LE, Siseles NO, Royer M, Prada $\mathrm{M}$, Sayegh F, et al. Metabolic syndrome throughout the menopausal transition: influence of age and menopausal status. Climacteric 2006; 9:40-8.

27. Rodrigues AD. Fatores associados à síndrome metabólica em mulheres no climatério em atendimento em ambulatório do sul do Brasil [Dissertação de Mestrado]. São Leopoldo: Programa de Pós-graduação em Saúde Coletiva, Universidade do Vale do Rio dos Sinos; 2011.

28. Feng Y, Hong X, Wilker E, Li Z, Zhang W, Jin D, et al. Effects of age at menarche, reproductive years, and menopause on metabolic risk factors for cardiovascular diseases. Atherosclerosis 2008; 196:590-7.

29. Nakazone MA, Pinheiro A, Braile MC, Pinhel MA, Sousa GF, Pinheiro Jr. S, et al. Prevalence of metabolic syndrome using NCEP-ATPIII and IDF definitions in Brazilian individuals. Rev Assoc Med Bras 2007; 53:407-13.
30. Santos AC, Ebrahim S, Barros H. Gender, socioeconomic status and metabolic syndrome in middle-aged and old adults. BMC Public Health 2008; 8:62.

31. Lovejoy JC, Champagne CM, de Jonge L, Xie H, Smith SR. Increased visceral fat and decreased energy expenditure during the menopausal transition. Int J Obes (Lond) 2008; 32:949-58.

32. Orsatti FL, Nahas EA, Nahas-Neto J, Maesta N, Padoani NP, Orsatti CL. Anthropometric measures: predictors of non-transmissible chronic diseases in postmenopausal women in the Southeast region of Brazil. Rev Bras Ginecol Obstet 2008; 30:182-9.

33. Eshtiaghi R, Esteghamati A, Nakhjavani M. Menopause is an independent predictor of metabolic syndrome in Iranian women. Maturitas 2010; 65:262-6.

34. Ghosh A. Comparison of risk variables associated with the metabolic syndrome in pre- and postmenopausal Bengalee women. Cardiovasc J Afr 2008; 19:183-7.

35. Henneman P, Janssens AC, Zillikens MC, Frolich M, Frants RR, Oostra BA, et al. Menopause impacts the relation of plasma adiponectin levels with the metabolic syndrome. J Intern Med 2010; 267:402-9.

36. Lewington S, Clarke R, Qizilbash N, Peto R, Collins R. Age-specific relevance of usual blood pressure to vascular mortality: a meta-analysis of individual data for one million adults in 61 prospective studies. Lancet 2002; 360:1903-13.

37. Sociedade Brasileira de Hipertensão; Sociedade Brasileira de Cardiologia; Sociedade Brasileira de Nefrologia. V diretrizes brasileiras de hipertensão arterial. Arq Bras Cardiol 2007; 89:e24-e79.

38. Sieminska L, Wojciechowska C, Foltyn W, Kajdaniuk D, Kos-Kudla B, Marek B, et al. The relation of serum adiponectin and leptin levels to metabolic syndrome in women before and after the menopause. Endokrynol Pol 2006; 57:15-22.

39. Reaven G. Why a cluster is truly a cluster: insulin resistance and cardiovascular disease. Clin Chem 2008; 54:785-7.

40. Sociedade Brasileira de Diabetes. Tratamento e acompanhamento do diabetes mellitus. Diretrizes da Sociedade Brasileira de Diabetes. Rio de Janeiro: Sociedade Brasileira de Diabetes; 2007.

41. Ferin M, Jewelewicz R, Warren M. The menstrual cycle: physiology, reproductive disorders and infertility. Oxford: Oxford University Press; 1993.

42. Lejskova M, Alusik S, Suchanek M, Zecova S, Pitha J. Menopause: clustering of metabolic syndrome components and population changes in insulin resistance. Climacteric 2011; 14:83-91.

Recebido em 04/Jan/2012

Versão final reapresentada em 11/Abr/2012

Aprovado em 19/Abr/2012 